\title{
Predictive analysis of optical ablation in several dermatological tumoral tissues
}

\author{
F. Fanjul-Vélez , A. Blanco-Gutiérrez, I. Salas-García, N. Ortega-Quijano, J. L. Arce-Diego \\ Applied Optical Techniques Group, TEISA Department, University of Cantabria \\ Av. de los Castros s/n, 39005 Santander (Spain)
}

\begin{abstract}
Optical techniques for treatment and characterization of biological tissues are revolutionizing several branches of medical praxis, for example in ophthalmology or dermatology. The non-invasive, non-contact and non-ionizing character of optical radiation makes it specially suitable for these applications. Optical radiation can be employed in medical ablation applications, either for tissue resection or surgery. Optical ablation may provide a controlled and clean cut on a biological tissue. This is particularly relevant in tumoral tissue resection, where a small amount of cancerous cells could make the tumor appear again. A very important aspect of tissue optical ablation is then the estimation of the affected volume. In this work we propose a complete predictive model of tissue ablation that provides an estimation of the resected volume. The model is based on a Monte Carlo approach for the optical propagation of radiation inside the tissue, and a blow-off model for tissue ablation. This model is applied to several types of dermatological tumoral tissues, specifically squamous cells, basocellular and infiltrative carcinomas. The parameters of the optical source are varied and the estimated resected volume is calculated. The results for the different tumor types are presented and compared. This model can be used for surgical planning, in order to assure the complete resection of the tumoral tissue.
\end{abstract}

Keywords: laser-tissue interaction, optical ablation, optical propagation model, Monte Carlo method

\section{INTRODUCTION}

The use of biomedical optics in clinical praxis is revolutionizing diagnostic, treatment and surgical applications [1]. Optical techniques employ non-invasive, non-contact and non-ionizing radiation, with a high degree of spatial control of the spot. These features are crucial for an effective and harmless medical device. Optical diagnosis benefits from high resolution, high-contrast images, while optical treatment provides a selective and controlled therapeutical procedure that minimizes collateral thermal damage. Optical surgery is also a quite extended medical procedure, either for tissue resection or as an optical scalpel [2].

The use of optical radiation for malignant biological tissue resection provides a more precise result than conventional surgery. This means fewer complications for the patient, such as sepsis or tumor recurrence. This technique can be applied either externally or internally in a minimally invasive way by means of endoscopes [1]. The advantages of optical ablation are fully developed by means of a surgical planning tool for obtaining an efficient result on the patient. This predictive model of optical ablation is highly desired specially when applied to tumor resection. In this case the complete elimination of the tumoral tissue is needed in order to prevent tumor recurrence. This predictive tool should be able to provide an estimation of the ablated volume as a function of the optical source, the tumor type and geometry, and the exposition time, among others. This is particularly relevant in dermatology for the elimination of different types of non-melanoma skin cancer [3].

In this work we present a predictive model of optical ablation in biological tissues. Section 2 presents the fundamentals of the model, including light propagation in turbid media, as long as the specific ablation approach. Section 3 describes the three types of non-melanoma skin cancer that will be analysed. Section 4 contains the results of the predictive model applied to non-melanoma skin cancer for different optical source parameters. Finally section 5 includes the conclusions of the work.

*fanjulf@unican.es; phone +34 9422067 30; fax +34 9422018 73; www.teisa.unican.es/toa

Medical Laser Applications and Laser-Tissue Interactions VI, edited by Lothar D. Lilge, Ronald Sroka,

Proc. of OSA Biomedical Optics-SPIE Vol. 8803, 88030R · (C) 2013 OSA-SPIE

CCC code: $1605-7422 / 13 / \$ 18 \cdot$ doi: $10.1117 / 12.2031948$ 


\section{PREDICTIVE MODEL OF OPTICAL ABLATION}

Optical ablation of biological tissues is a complex procedure than can be based on several ablation mechanisms [2]. This process involves, among others, thermal denaturation or even photochemical effects [4]. In order to model this complex procedure, first of all it is necessary to know the spatial optical radiation distribution in the biological tissue. This information will provide the basics for energy deposition and allow a further prediction of the possible ablation effect.

\subsection{Optical propagation model}

Light propagation in biological tissues can be modeled in different ways. Modeling a biological tissue implies dealing with an heterogeneous medium, which does not usually allow an analytical exact approach of the radiation pattern with Maxwell equations. For the problem we are dealing with, the distribution of light in a three-dimensional tissue must be obtained. This objective is reached by means of the Radiation Transport Theory (RTT) [1]. The model assumes that the scattering events are sufficiently numerous as to the light to be considered incoherent, in such a way that polarization or interference effects can be neglected. As a consequence, the basic parameter of light is the specific intensity, $I(r, \hat{s})$, that is, the light power per unit area per unit solid angle. The radiation is expected to be at the point $\vec{r}$, and to follow the direction $\hat{s}$. The scattering events are treated according to the scattering phase function, $p\left(\hat{s} \cdot \hat{s}^{\prime}\right)$, which contains the probabilities of light to be scattered in the different directions. Optical radiation comes from direction $\hat{s}^{\prime}$ and is redirected to $\hat{s}$. The basic idea in order to write the differential radiation transport equation is that radiation from a particle attenuates due to absorption and scattering and also gains power because another particle can scatter light in the direction of the particle of interest. If there are no sources inside the tissue and for a steady-state situation, this can be written as

$$
\hat{s} \cdot \bar{\nabla} I(r, \hat{s})=-\left(\mu_{a}+\mu_{s}\right) I(r, \hat{s})+\frac{\mu_{s}}{4 \pi} \int_{4 \pi} p\left(\hat{s} \cdot \hat{s}^{\prime}\right) I\left(r, \hat{s}^{\prime}\right) d \Omega^{\prime}
$$

An analytical solution can be given for this equation. The main attempts try to simplify the problem by making approaches in limiting cases, such as absorption-dominant limit or diffusion approximation, depending on the relative importance of absorption and scattering events in a particular biological tissue. However, there is another possibility to solve the equation, using a numerical approximation. Numerical analysis has been widely applied to a great variety of problems governed by differential equations. In the particular topic of the radiation transport equation, the Monte Carlo method has demonstrated its applicability and accuracy, compared with exact solutions. Perhaps the most used implementation of the Monte Carlo method applied to the RTT model is the one by Wang and Jacques [5]. They programmed the Monte Carlo method in standard C. The key point is the inclusion of the random character on a computer, by means of a mathematical probability analysis, in such a way that numbers with any probability distribution can be obtained from numbers that follow a uniform distribution between 0 and 1 . Light is treated as a sequence of photons, whose number is intended to be representative of the accuracy of the solution obtained. One photon is launched and its trajectory, affected by scattering, and loose of energy are calculated, while the absorption in each point is stored. Cylindrical symmetry is assumed, because laser beams usually show this kind of symmetry, so in fact the data can be interpreted as coming from a 3-D analysis. The complete tissue is divided in a two-dimensional grid in the $\mathrm{r}$ and $\mathrm{z}$ directions. As usual, more accurate results require a smaller grid, but the need of a reduced time of computation imposes a limit.This implementation of the Monte Carlo model is also multi-layered, so it is possible to define several layers of different materials, with their borders always perpendicular to the laser beam, as it is usually the case in biological tissues.

For the appropriate definition of the model, the characteristics and dimensions of each layer are required. The optical parameters needed are the index of refraction, $n$, the absorption coefficient, $\mu_{a}$, the scattering coefficient, $\mu_{s}$, and the anisotropy of scattering, $g$. This last parameter is called average cosine of scatter (dimensionless), and is related with the scattering phase function. The average cosine of scatter gives an idea about the probability of being scattered in a particular direction: 


$$
g=\frac{\int_{4 \pi} p\left(\hat{s} \cdot \hat{s}^{\prime}\right) \hat{s} \cdot \hat{s}^{\prime} d \Omega^{\prime}}{\int_{4 \pi} p\left(\hat{s} \cdot \hat{s}^{\prime}\right) d \Omega^{\prime}}=\frac{1}{4 \pi W_{o}} \int_{4 \pi} p\left(\hat{s} \cdot \hat{s}^{\prime}\right) \hat{s} \cdot \hat{s}^{\prime} d \Omega^{\prime}=\frac{1}{2 W_{o}} \int_{4 \pi} p(\cos \theta) \cos \theta \sin \theta d \theta
$$

where the albedo is defined by

$$
W_{o}=\frac{1}{4 \pi} \int_{4 \pi} p\left(\hat{s} \cdot \hat{s}^{\prime}\right) d \Omega^{\prime}=\frac{\sigma_{s}}{\sigma_{a}+\sigma_{s}}=\frac{\mu_{s}}{\mu_{a}+\mu_{s}} .
$$

The average cosine of scatter gives an idea about the probability of being scattered in a particular direction. For instance, $g=0$ implies that all directions all equally probable. If $g>0$ the radiation tends to be scattered forward, and vice versa. The albedo tries to illustrate the predominance of absorption or scattering in a particular tissue.

\subsection{Ablation model}

The process of ablation is intrinsically complex to model, as it involves also mechanical changes in the tissue structure. A complete model should take into account, among others, the mechanical properties of the biological tissue, the main mechanism of laser-tissue ablation, thermal denaturation, phase transitions or dynamical optical properties [4]. Most of these properties depend at the same time on the optical source parameters. Usual models are not capable of considering all these parameters. There are several approaches to model the ablation process, from mechanistic models such as the thermo-mechanical or the stationary state vaporization model, to heuristic models such as the blow-off or stationary state models [4]. The mechanistic models try to take into account the relationship between tissue and laser parameters, at least to some extent. The complexity of this type of models is high. They were first simplified by neglecting the explosive nature of ablation. The ablation process was then explained by a vaporization process, in the so-called stationary state vaporization models. The assumptions of these models, based on metal ablation, provide an erroneous estimation of the temperature distribution inside the tissue. The thermomechanical models consider a double phase transition in the tissue, from solid to liquid, and from liquid to vapor. In this way material ejection by ablation is explained. In general the assumptions of the model are more valid for gelatin-like tissues, as thermal denaturation produces a liquid phase in the biological ablated tissue prior to ejection.

Heuristic models constitute a more phenomenological approach to the study of optical ablation. Steady-state heuristic models are valid for pulsed laser sources with pulse duration of around microseconds. In these optical sources material removal is usually concurrent with optical irradiation. An energy density threshold is assumed for optical ablation to begin, shortly after irradiation. The process of material removal is thought to be continuous and will depend on the exposition time of the optical source. In this way, as the irradiation time increases, so grows the depth of the removal tissue procedure. The assumption of a quick beginning of tissue removal makes the model appropriate only for pulse durations of microseconds or more, and with irradiations over the threshold.

Another heuristic model is the blow-off model. This approach establishes that there is a finite radiant exposure threshold for beginning the ablation procedure. If radiation is below this threshold, there will be thermal interaction but no ablation. Material removal is assumed to begin after finishing the irradiation. These assumptions make thermal confinement to be required [4]. In general these conditions are fulfilled if pulse duration is below $100 \mathrm{~ns}$. In the particular case of employing the simple Beer-Lambert law for optical propagation, it is possible to express ablation depth $\delta$ as:

$$
\delta=\frac{1}{\mu_{\mathrm{a}}} \ln \left(\frac{\phi_{0}}{\phi_{\mathrm{th}}}\right)
$$

Where $\varphi_{0}$ and $\varphi_{t h}$ are the initial and threshold radiation, respectively. The effect of scattering is neglected in this approach.

In this work we use a predictive ablation model based on the blow-off approach for non-melanoma skin cancer removal applications. We combine the Monte Carlo approach with this heuristic model in order to obtain a reasonable predictive tool in tumoral tissue ablation in dermatology for several types of lesions. 


\section{NON-MELANOMA SKIN CANCER TUMORS}

Nonmelanoma skin cancer is one of the main health problems in western countries. One third of the diagnosed cancers are of this type, and it is the most frequent malignant neoplasia in humans [6]. The origin of this cancer is epithelial and includes $80 \%$ of cutaneous cancers. In this way, they appear 20 times more than the melanoma. Nonmelanoma skin cancer includes basocellular carcinoma or squamous cell carcinoma, among others. The former appears in the basal epidermal cells and it is originated without presenting a previous lesion. Metastasis risk is extremely low, but this kind of cancer is able to provoke great local tissue destruction. An example of basocellular carcinoma can be seen in Figure 1. Squamous cell carcinoma can be basal or spinous, presents a fast growing process and it is more likely to invade other tissues. Previous lesions that degenerate in squamous cell carcinoma are actinic keratosis and Bowen disease [6]. Actinic keratosis is the most frequent premalignant lesion, with an apparition rate ten times over that of the basocellular carcinoma. It is the main lesion that acts as a precursor of squamous cell carcinoma. Squamous cell carcinoma provokes metastasis in $5 \%$ of the cases, due to linfatic or vascular infiltration.

Figure 1. Superficial basocellular carcinoma.

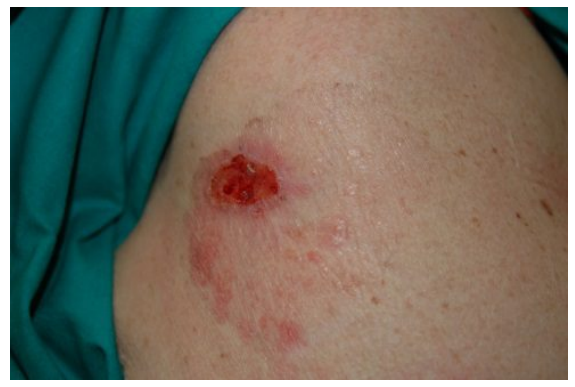

The origin of nonmelanoma skin cancer is greatly varying, but the main present cause is the chronic exposition to ultraviolet radiation. These cases are daily seen in medical praxis, either in the primary service or by the specialist. The incidence of nonmelanoma skin cancer is increasing by $4 \%$ each year. Nowadays a white male has a $33 \%$ risk of developing a basocellular carcinoma and more than $9 \%$ of suffering from a squamous cell carcinoma. This great increase in the incidence is thought to be related with some habits like the rising amount of solar exposition, the change in clothing habits that let more skin exposed to solar radiation, the increased longevity and the reduction of ozone in the atmosphere. The fact that the most usual areas affected are the exposed body parts like the face, and the low death rate of nonmelanoma skin cancer make cure and cosmetic the principal objectives of the treatment. In this way, the treatment must be oriented to tumour elimination, relapse avoidance, recovery of the cosmetic and functional appearance of skin and maximization of the rate cost-effectiveness.

In this work we will apply the predictive model to three types of non-melanoma skin cancer. We will now describe slightly each one. Squamous Cell Carcinoma (SCC) is a type of cancer that appears in the epidermis of skin, but also in the digestive or pulmonary epithelium. It is the second most common cancer type. It is usually successfully treated by surgical removal. However, some aggressive cases provoke extended tissue destruction and metastasis, and finally death. Basocellular Carcinoma (BCC) is the most common type of cancer. It appears in basal cells, which are present in the deepest epidermis. The origin of this cancer is usually solar exposition, and as a consequence it is normally located in the face, ears, neck, head, shoulders or back. Sometimes it may also appear in non-exposed areas. Even radiation exposition, recursive open wounds, burns or infections are in the origin of this cancer. The last one, Infiltrative Carcinoma (INF) is the most invasive one. This cancer grows over the cell layer where it first appeared. It is common in mammalian cancer.

\section{APPLICATION OF THE PREDICTIVE MODEL TO DERMATOLOGICAL TUMORS}

The application of a predictive analysis of tissue ablation to non-melanoma skin cancer requires an appropriate choice of the model employed. Optical propagation was modeled by a Monte Carlo method. Tissue ablation was predicted by means of a blow-off based approach, due to the fact that laser pulses of less than 100 ns could be employed in nonmelanoma skin cancer applications. The model presented in previous sections was applied to three different types of nonmelanoma skin cancer: squamous cells carcinoma, basocellular carcinoma and infiltrative carcinoma. Every tumor type presents differentiated characteristics that also affect optical properties [3]. First we applied the Monte Carlo method with a particular tumor depth taking into account the optical properties of the carcinoma type, and specific parameters for 
the optical source, mainly wavelength, irradiance, exposition time, spatial profile and radius. The wavelength was fixed at $633 \mathrm{~nm}$, so the optical properties were taken accordingly [3,8]. The optical properties of healthy skin appear in Table 1.

Table 1. Healthy skin optical and geometrical properties [8].

\begin{tabular}{|l|c|c|c|c|c|}
\hline \multicolumn{1}{|c|}{ Layer } & $\mathbf{n}$ & $\boldsymbol{\mu}_{\mathbf{a}}[\mathbf{1} / \mathbf{c m}]$ & $\boldsymbol{\mu}_{\mathbf{s}}[\mathbf{1} / \mathbf{c m}]$ & $\mathbf{g}$ & $\mathbf{d}[\mathbf{c m}]$ \\
\hline Epidermis & 1.5 & 25 & 480 & 0.79 & 0.0065 \\
\hline Upper dermis & 1.4 & 2.7 & 187 & 0.82 & 0.063 \\
\hline Blood plexus & 1.35 & 25 & 400 & 0.98 & 0.072 \\
\hline Lower dermis & 1.4 & 2.7 & 187 & 0.82 & 0.1285 \\
\hline $\begin{array}{l}\text { Subcutaneous } \\
\text { fat }\end{array}$ & 1.45 & 0.2 & 20 & 0.8 & 0.1605 \\
\hline Vessel wall & 1.37 & 6 & 414 & 0.91 & 0.2215 \\
\hline Blood & 1.35 & 25 & 400 & 0.98 & 0.8595 \\
\hline
\end{tabular}

Each tumor was supposed to be located at skin surface, with a depth of $0.3 \mathrm{~cm}$, near the limit of optical penetration depth. Optical properties of the tumors were obtained from [3]. After the tumor healthy skin tissue was assumed, following the previous layered analysis. We considered optical source radii of $1 \mathrm{~cm}$ and $1 \mathrm{~mm}$, different irradiances and two possible spatial profiles: Gaussian and cylindrical. Examples of the results of optical absorption in non-melanoma skin cancer can be seen in Figure 2.

Figure 2. Optical absorption for a Gaussian optical source of $1 \mathrm{~mm}$, total delivered energy of $10 \mathrm{~J}$, and SCC (left) and BCC (right) tumors.
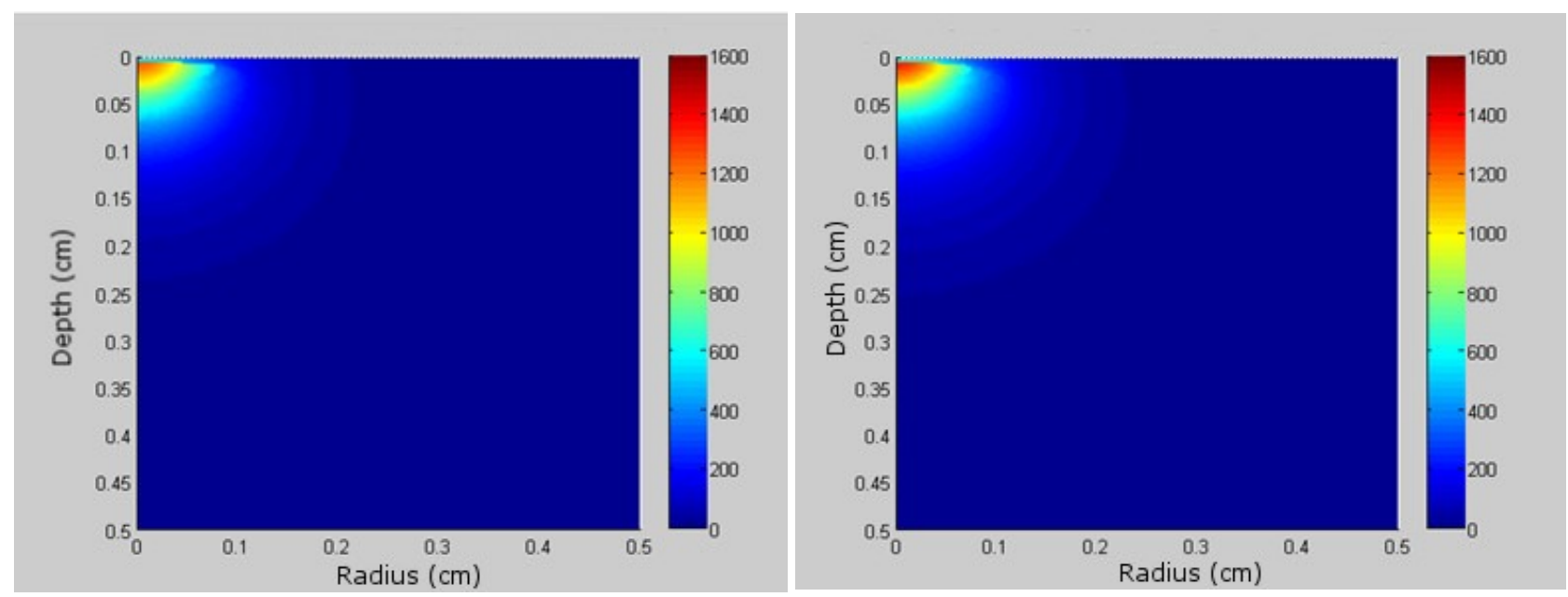

In this figure the optical irradiation comes from above and impinges at the upper left corner. This corner coincides with the laser beam center. As it can be seen the optical distribution is preferentially concentrated near the tissue surface, near the laser beam impact. It decreases quickly both in depth and in the radial direction. A slight difference between both tumors can be appreciated, with a bigger penetration depth in the case of a BCC tumor.

Afterwards the spatial distribution allowed us to apply the blow-off model according to the previous considerations. The threshold for ablation, needed for the model, is taken in this case from that for collagen tissues [7], as the composition of skin is greatly similar to that. As this value depends on pulse duration, we chose 5 ps pulses, in order to cope with the assumptions of the heuristic approach, particularly due to thermal confinement. The obtained value is about $5 \mathrm{~J} / \mathrm{cm} 2$. 
With all these data we made several simulations of healthy skin and skin affected by different tumor types in order to estimate the ablated volume. First we made a prediction of the ablated area for a $1 \mathrm{~cm}$ radius Gaussian laser beam at 633 $\mathrm{nm}$, for different total energies. Some results appear in Figure 3.

Figure 3. Ablated area (red) of a $1 \mathrm{~cm}$ radius Gaussian beam at $633 \mathrm{~nm}$ for $5 \mathrm{~J}$ and SCC tumor (upper left), $16 \mathrm{~J}$ and SCC tumor (upper right), $5 \mathrm{~J}$ and $\mathrm{BCC}$ tumor (lower left) and $16 \mathrm{~J}$ and $\mathrm{BCC}$ tumor (lower right).
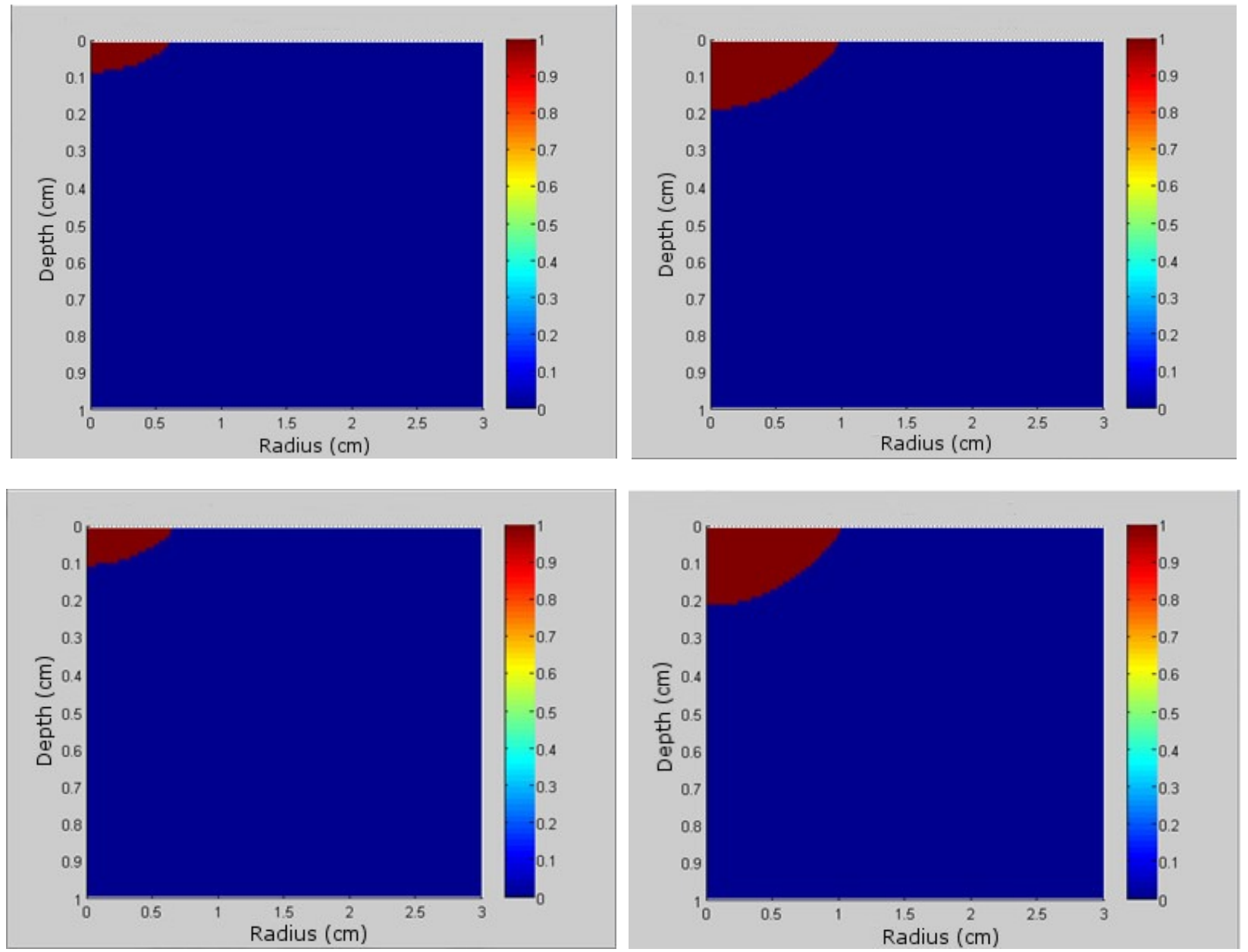

As it can be clearly seen, the ablated area follows, as expected, the optical irradiation distribution. The Gaussian profile of the optical beam delineates the general geometry of the ablation, whose maximum depth is located at the beam center. The increase of total delivered energy makes the ablated area bigger, both in radius and in depth. Regarding the differences between both tumors, SCC and BCC, the ablation depth for the latter is larger for both energies. This fact justifies the importance of the predictive analysis, as tumoral tissue resection would require a lesion-dependent planning.

If treatment planning is intended, the information contained in previous figures could be not enough for the practitioner. Graphical data coming from superficial or volumetric ablation are preferred, in order to try to better predict if the ablated volume will last for the application. Figure 4 shows superficial and volumetric images of the ablated surface or volume, respectively, for different cases. The laser beam has a $1 \mathrm{~cm}$ radius and a Gaussian spatial profile. After a total delivery of $100 \mathrm{~J}$, results are shown both for Infiltrative tumor and for healthy skin. 
Figure 4. Superficial (upper graphs) and volumetric (lower graphs) ablated areas and volumes, respectively, for a Gaussian 1 $\mathrm{cm}$ laser beam, after a total delivery of $100 \mathrm{~J}$, for Infiltrative tumor (left) and healthy skin (right).
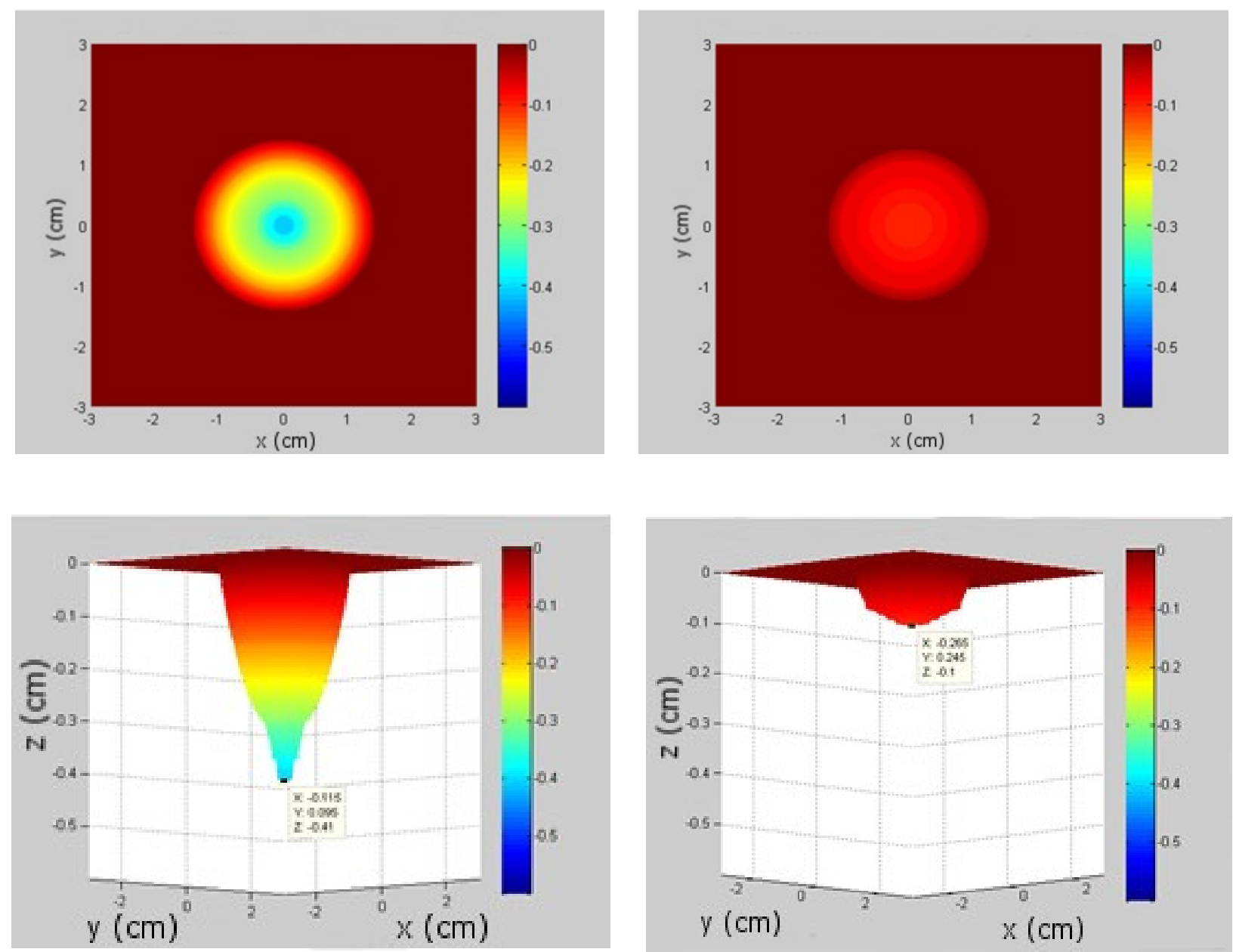

The differences between Infiltrative carcinoma and healthy skin are evident from the figure. With the same optical source, and under the same irradiance conditions, the ablated volume is much bigger in the case of Infiltrative carcinoma. The ablation depth can go even to $4 \mathrm{~mm}$ in this case, while it would remain at around $1 \mathrm{~mm}$ for healthy skin.

As a consequence, the predictive analysis exposed makes it possible to predict the ablated volume and to control it by changing the optical parameters of the beam, taking into account the particular lesion we are dealing with. This model could be used for optical surgery planning in tumoral tissue resection.

\section{CONCLUSIONS}

In this work a predictive model of optical biological tissue ablation has been presented. The model is based on a Monte Carlo approach for light propagation in turbid media and a blow-off approach for the ablation process. The predictive model is applied to three different types of non-melanoma skin cancer, as a function of optical source parameters, mainly wavelength, irradiance, spatial profile, beam radius and exposition time. The results are presented as 3D volumetric ablation zones, and compared for the different lesions, beam radii, irradiances and exposition times. This model could be useful for medical surgical treatment planning in medical praxis, specially for tumor resection, in order to avoid recurrence. 


\section{ACKNOWLEDGEMENTS}

This work has been partially supported by the project MAT2012-38664-C02-01 of the Spanish Ministery of Economy and Competitiveness, and by the San Cándido Foundation.

\section{REFERENCES}

[1] Vo-Dinh, T., [Biomedical Photonics Handbook], CRC Press, Boca Raton (2003).

[2] Niemz, M. H., [Laser-Tissue Interactions Fundamentals and Applications], Springer, Germany (2004).

[3] Salas-García, I., Fanjul-Vélez, F. and Arce-Diego, J. L., "Influence of the human skin tumor type in Photodynamic Therapy analysed by a predictive model", International Journal of Photoenergy 2012, 1-9 (2012).

[4] Vogel, A. and Venugopalan, V., "Mechanisms of Pulsed Laser Ablation of Biological Tissues", Chemical Reviews 103, 577-644 (2003).

[5] Wang, L., Jacques, S. L. and Zheng, L., "MCML - Monte Carlo modeling of light transport in multi-layered tissues", Computer methods and programs in biomedicine 47, 131-146 (1995).

[6] Diepgen, T. L. and al., "The Epidemiology of Skin Cancer", British Journal of Dermatology 146(61), 1-6 (2002).

[7] Loesel, F. H., Niemz, M. H., Bille, J. F. and Juhasz, T., "Laser-Induced Optical Breakdown on Hard and Soft Tissues and Its Dependence on the Pulse Duration: Experiment and Model”, IEEE J Quantum Electronics 32, 1717-1722 (1996).

[8] Tuchin, V. V., "Laser light scattering in biomedical diagnostics and therapy", Journal of Laser Applications 5, 43-60 (1993). 